\title{
Irreproducible positive results on the Cobas AmpliPrep/ Cobas TaqMan HIV-1 Qual test are qualitatively different from confirmed positive results
}

\author{
by \\ Jean Maritz
}

Thesis presented in fulfilment of the requirements for the degree of Master of Medicine (Virological Pathology) in the Faculty of Medicine and Health Sciences at Stellenbosch University

Supervisor: Dr. Gert Uves van Zyl

December 2013 


\section{Declaration}

I, the undersigned, hereby declare that the work contained in this assignment is my original work and that it has not previously in its entirety or in part been submitted at any university for a degree.

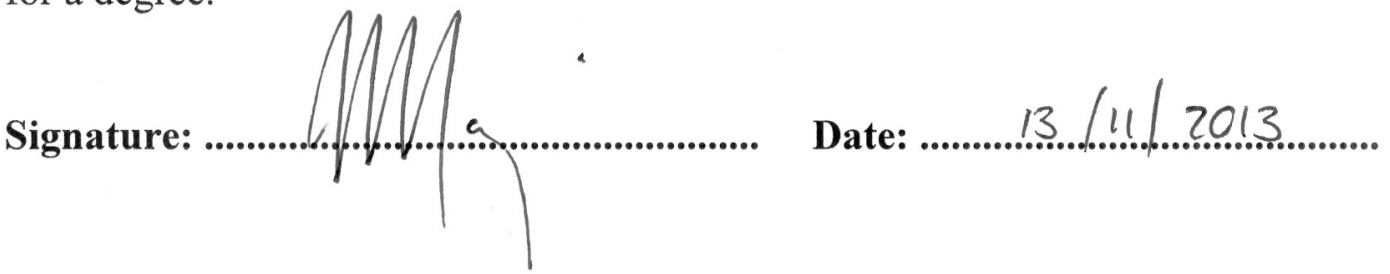


This is the pre-peer reviewed version of the following article: Maritz, J., van Zyl, G. U. and Preiser, W. (2013), Irreproducible positive results on the Cobas AmpliPrep/Cobas TaqMan HIV-1 Qual test are different qualitatively from confirmed positive results. J. Med. Virol..

doi: 10.1002/jmv.23811, which has been published in final form at

http://onlinelibrary.wiley.com.ez.sun.ac.za/doi/10.1002/jmv.23811/abstract. 
Irreproducible positive results on the Cobas AmpliPrep/ Cobas TaqMan HIV-1 Qual test are qualitatively different from confirmed positive results

Jean Maritz ${ }^{1}$ *

Gert U van Zyl ${ }^{1}$

Wolfgang Preiser ${ }^{1}$

${ }^{1}$ Division of Medical Virology, Department of Pathology, Stellenbosch University Faculty of Medicine and Health Sciences and National Health Laboratory Service, Cape Town, South Africa

Institution at which work was conducted:

Division of Medical Virology

Department of Pathology, Faculty of Medicine and Health Sciences

Stellenbosch University and National Health Laboratory Service Tygerberg

PO Box 19063, Tygerberg 7505, South Africa

${ }^{*}$ Correspondence to: Jean Maritz, Division of Medical Virology, Department of Pathology,

Stellenbosch University Faculty of Medicine and Health Sciences and National Health Laboratory

Service, PO Box 19063, Tygerberg 7505, South Africa

Telephone: +27219389354

Fax: $\quad+27219389361$

E-mail: $\quad$ maritzj@sun.ac.za 


\section{Abstract}

Criteria that define low positive results on the COBAS $^{\circledR}$ AmpliPrep/COBAS ${ }^{\circledR}$ TaqMan (CAP/CTM) HIV1 Qual test as inconclusive have been adopted by all academic centres in South Africa that conduct infant HIV PCR, following previous investigations that showed poor specificity of these results. Retesting all these specimens has considerable cost implications. It was therefore attempted to better characterise such inconclusive results, by comparing those that prove to be either negative or positive on follow-up testing. This retrospective, laboratory-based study found that 193 of 211 (91.5\%) patients with with previous inconclusive results (defined as reported positive by CAP/CTM but with cycle threshold [Ct] values of $>32$ and/or fluorescence intensity $[\mathrm{FI}]$ values of $<5$ ) tested negative and only 18 (8.5\%) positive using independently obtained follow-up samples after a median of 28 days). The only significant independent predictor of a later positive result was a higher FI value (3.326 vs. $0.495, p<0.0001$ ), whereas $\mathrm{Ct}$ values were not independently predictive. Specimens from patients negative on follow-up testing were qualitatively different from specimens that proved to be true positive. As the lower FI values in false-positive compared to true-positive results are probably indicative of a non-specific signal, the incorporation of stringent amplification slope criteria in the assay's test definition file may improve correct classification and thus reduce the need for repeat testing of a large number of inconclusive specimens.

\section{Key words:}

PCR

False positive

Infant diagnosis 


\section{Introduction}

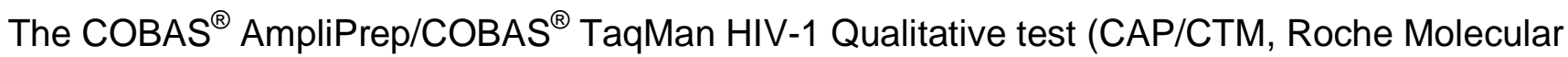
Systems Inc., Branchburg, NJ) is a total nucleic acid real-time polymerase chain reaction (PCR) assay that detects HIV-1 proviral DNA and HIV-1 RNA (Roche, 2007). The assay is well suited for early infant diagnostic testing in the South African context as it is a high-throughput, automated system, optimised to yield comparable amplification efficiencies for different HIV-1 group M subtypes and can test both whole EDTA blood and dried blood spot (DBS) samples. However, at the time of writing, the assay is classified as a "Research Use Only" assay without FDA approval or CE marking (Roche, 2007).

As early antiretroviral therapy (ART) is proven to reduce HIV disease progression and early infant mortality by $75 \%$ and $76 \%$ respectively (Violari et al., 2008), current South African guidelines advise not to delay the initiation of ART in young children with a positive HIV PCR while awaiting the result of the baseline HIV-1 viral load test which also serves as confirmation (National Department of Health, 2010). In a high burden, limited resource setting like South Africa, reliable early diagnosis of infant HIV-1 infection with a single screening PCR is crucial as awaiting confirmatory test results can introduce treatment delays, especially in rural regions with poor access to healthcare. In 2011, between 248,869 and 284,023 HIV-exposed infants were born in South Africa (Sherman, 2012); this is in stark contrast to a resource rich setting like Canada, where the CAP/CTM assay is also used for infant diagnosis (Ontario province, [Public Health Ontario, 2011]) but as few as four infants were diagnosed with HIV infection countrywide in 2008 (Public Health Agency Canada, 2010). Settings like South Africa are not afforded the luxury of comprehensive diagnostic and confirmatory algorithms for a large part of the population, and false-positive screening HIV PCR results can thus pose a significant problem resulting in unnecessary exposure to ART, a waste of resources and diagnostic challenges later in life (Zanchetta et al., 2008). 
With the decrease in recent years in the prevalence of infants who acquire HIV through mother-tochild transmission (Moodley et al., 2013), diagnostic assays now require very high specificity to maintain a high positive predictive value. Previously, we reported poor specificity (97.1\%) and positive predictive value (78.7\%) of the CAP/CTM in testing DBS for HIV-1 infection compared to the reference method, defined as two AMPLICOR ${ }^{\circledR}$ HIV-1 DNA test v1.5 (Roche ${ }^{\circledR}$ Molecular Systems Inc., Branchburg, NJ) (“Amplicor") results (Maritz et al., 2012). We concluded that the observed decreased specificity was unlikely to be due to superior sensitivity of the CAP/CTM but rather due to non-specific test signals, as results were not repeatable using a second DBS. Furthermore, the amplification curves of discordant (i.e. CAP/CTM positive, Amplicor negative) samples showed significantly lower fluorescence intensity $(\mathrm{FI})$ and significantly higher cycle threshold $(\mathrm{Ct})$ values compared to concordant positive samples. The low positive predictive value of the CAP/CTM was subsequently confirmed by another South African study (Feucht et al., 2012).

To safeguard against the reporting of false positive results, we have, based on the abovementioned comparison, defined a set of criteria for the diagnosis of HIV-1 infection based on $\mathrm{FI}$ and $\mathrm{Ct}$ values as reported by the CAP/CTM system's Amplilink ${ }^{\circledR}$ software, to allow objective evaluation of the real-time curve measurements by different users. Subsequently all academic centres in South Africa have adopted these or similar criteria for result interpretation: in cases with absolute FI values below 5 and/or Ct values higher than 32, results are reported as "inconclusive" and a follow-up sample to be obtained 10 to 14 days later is requested. In this study, the characteristics of samples with such inconclusive results that were followed up by testing a second, independently obtained sample are described. 


\section{Materials and Methods}

Objectives. To ascertain the true HIV status of samples yielding inconclusive results on the CAP/CTM, as defined by abovementioned criteria, through routine follow-up sample testing. It was further aimed to better characterise initially inconclusive CAP/CTM results by comparing $\mathrm{FI}$ and $\mathrm{Ct}$ values between samples that were proven to be either positive or negative on the follow-up specimen.

Study design. This retrospective, laboratory-based study was performed at the Division of Medical Virology, National Health Laboratory Service Tygerberg, and was approved by the Human Research Ethics Committee of the University of Stellenbosch (approval number N11/09/283).

Inclusion criteria. Patients under the age of two years with two or more blood samples sent for HIV-1 PCR testing between January 2009 and May 2011, who had an initial inconclusive CAP/CTM result and a definitive result on follow-up. Eligible sample types included DBS and whole EDTA blood, as advised by the manufacturer (Roche, 2007), and as per laboratory standard operating procedure, EDTA samples older than five days were not tested.

Exclusion criteria. Samples with measurable $\mathrm{Fl}$ and $\mathrm{Ct}$ values, but that were reported as negative by the instrument, the significance of which is unknown (Maritz et al., 2012), and samples from which the hospital number, name or date of birth could not be matched.

Sample selection. Data was extracted from the Laboratory Information System (LIS) of all samples, sent for diagnostic HIV PCR tests, between 1 January 2009 and 31 May 2011 in order to identify patients from whom more than one sample was sent. After extraction of the data, consecutive samples were identified by confirming surname, name, date of birth, and hospital number combinations of possible candidates. Patients who could not be matched due to missing data were excluded from the analysis. 
Amplification curve characteristics of the CAP/CTM assay. As CAP/CTM measurement values itself are not routinely captured on the LIS, selected user-accessible parameters are routinely exported after each PCR run and archived. These measurements include sample laboratory numbers and FI and Ct values and were merged into a single database for this study using software macros to avoid possible transcription errors. The resulting database was merged with the patient database described above using software macros and using laboratory numbers as identifiers. After matching the LIS records with CAP/CTM records, all patient identifiers were deleted and unique identifiers were assigned to each sample pair.

Definitions. A negative HIV PCR was defined by a "Target not detected" report from the CAP/CTM, and a definite positive HIV PCR result was defined by a "1 - Positive" report from the CAP/CTM with a FI value of at least equal to five and a Ct value lower than or equal to 32 as per laboratory standard operating procedure (SOP). Samples with a "1 - Positive" report from the CAP/CTM with a FI value of less than five and / or a Ct value higher than 32 were reported as "inconclusive". For the purposes of this study, inconclusive samples from patients who eventually tested positive are referred to as "inconclusive-positive", and inconclusive samples from patients who eventually tested negative are referred to "inconclusive-negative".

Statistics. Descriptive statistics, Mann-Whitney U-tests and logistic regression analyses were performed. 


\section{Results}

Of a total of 211 sample sets, it was found that 193 inconclusive samples were negative on follow-up (91.5\%) and 18 positive (8.5\%, Table I, Figure I). A significantly lower median Ct value was observed for inconclusive-positive samples compared to inconclusive-negative samples; similarly, a significantly higher median FI value in inconclusive-positive samples compared to inconclusivenegative samples was demonstrated (Table I). There was no significant difference in median time intervals between initial inconclusive and follow-up samples between the two groups, nor was the age of the patient at the time of the initial inconclusive PCR significantly different between the two groups. The impact of sample type (DBS or whole EDTA blood) on initial- or follow-up results could not be determined as very few DBS samples were tested during this comparison (Table I).

When using both $\mathrm{Ct}$ and $\mathrm{Fl}$ in logistic regression models to predict a definitive result of initially inconclusive samples, the adjusted model showed that $\mathrm{Fl}$ is an independent significant predictor of follow-up sample result whereas $\mathrm{Ct}$ is no longer predictive when $\mathrm{FI}$ is included in the model (Table II). The fact that a high $\mathrm{Ct}$ value that predicts a false-positive result in a univariate model is no longer predictive when the FI value is included in the model, suggests that the amplification curves of falsepositive results are qualitatively different from those of true-positive results. Review of the amplification curves as reported by the CAP/CTM supports this suggestion, as the expected curve shape of an exponentially amplifying target is not evident in samples with low $\mathrm{Ct}$ values and low $\mathrm{FI}$ values (Figure II). It was further found that an $\mathrm{FI}$ value of equal to or greater than 2.98 would have a specificity of $98.9 \%$ and correctly predict the follow-up sample result in $95.8 \%$ of samples currently classified as "inconclusive". 


\section{Discussion}

This study aimed to ascertain the HIV status of samples yielding inconclusive initial results using the CAP/CTM by using the results obtained from follow-up samples, and to better characterise inconclusive CAP/CTM results. As results generated by routine diagnostic screening for HIV-1 infections and those of follow-up samples, where indicated, were evaluated retrospectively, it was not possible to compare CAP/CTM results to a gold standard.

Significant differences in the FI and Ct values between inconclusive samples that eventually tested positive and those that tested negative were observed. Importantly, it was found that $91.5 \%$ of CAP/CTM positive samples with a Ct value of greater than 32 and/or a FI value of lower than 5 tested negative on a follow-up sample taken at a median of 28 days later. In a univariate model a low $\mathrm{Ct}$ value predicted positivity of a follow-up sample, however Ct values were no longer predictive of true positivity when used in a combined model with FI values. High FI values remained strongly predictive of true positivity in a combined model. Taken together, this suggests that true positive samples have steeper amplification slopes, suggesting true amplification, whereas false positive results have flat slopes (Figure II).

The high rate of irreproducible positive results is alarming, especially considering that, had the previously formulated criteria not been applied, the false-positive result as reported by the CAP/CTM would have been accepted. This means that, in the absence of confirmatory test results (National Department of Health, 2010) during the period of study, $25 \%$ of patients commenced on ART based on the positive result would not have been infected with HIV. This emphasises the need to define and use an indeterminate zone for result reporting.

The findings of this study using predominantly EDTA samples are in support of the previous observation of false-positive result reporting from DBS. Similar observations have been made by others; a significantly increased frequency in detectable HIV-1 levels using the quantitative CAP/CTM 
HIV-1 version 1 assay (which uses a comparable assay design to the CAP/CTM qualitative test) was reported in previously consistently-suppressed patients compared to the Roche Amplicor Monitor version 1.5 assay, which the authors speculated could be due to spurious signals caused by artefacts (Lima et al., 2009). Also, a study from a large South African hospital reported false positive HIV PCR results although the exact number generated by the CAP/CTM was not reported (Feucht et al., 2012). The reasons for flat amplification slopes of the inconclusive-negative samples are unclear. Suppressed HIV replication as a result of exposure to prolonged nevirapine monotherapy as part of a PMTCT strategy has been considered as an explanation for negative follow-up PCRs after initial low positive results; however it is considered unlikely that a single antiretroviral agent would result in abortive HIV infection in such a large number of patients. At the time of writing no data supporting this hypothesis have been published. Studies evaluating the peri- and postnatal history of infants with inconclusive PCR results may prove informative, but such data was not available during this evaluation. The lower volume of blood in DBS samples should also not have contributed to falsenegative follow-up results in this study as none of the follow-up tests were performed from DBS. Limitations of this study include the inability to correlate concurrent RNA viral load or integrated provirus load with initial or follow-up samples and the lack of comparison of all results to a gold standard or consensus result. Secondly, it is not possible to control for factors such as variability in sample characteristics or performance of specific components of the CAP/CTM instrument which could have contributed to discordant results on repeat samples. Assay and internal controls for each run were valid and it is therefore presumed that the instrument and assay performed optimally.

As all assay runs including negative and positive controls were valid, and with more than $95 \%$ of all results being negative, low-level or intermittent contamination is an unlikely explanation. Carry-over contamination is regarded as unlikely as an evaluation of 40 random assay runs containing inconclusive samples included in this study did not reveal a spatial association with positive specimens in 33/40 runs, nor a temporal association. The manufacturer's instructions regarding 
sample preparation, sample loading and daily instrument decontamination were adhered to at all times, and amplicon contamination is eliminated by the use of uracil-N-glycosylase (Roche, 2007). All assay runs were performed by a small complement of experienced technologists trained by Roche in a single laboratory. The problem of inconclusive results is experienced in academic laboratories across South Africa, and as stated previously, all laboratories have adopted interpretation criteria for positive results. It is hard to believe that technical incompetence should account for similar findings across multiple laboratories.

The findings of this study confirm that a large proportion of patients with initial results defined as being inconclusive are in fact negative. Although the current strategy of repeat testing patients with initial inconclusive results has significant cost implications and other disadvantages, we do not suggest that the criteria for triggering repeat testing be relaxed. Using a lower FI value cut-off might make reporting of inconclusive results more specific but insufficient data is available to establish whether this would not result in a decreased sensitivity. We believe that the only sound approach is to validate the test definition criteria of the assay, using raw amplification data, against a large data-bank of specimens that include inconclusive results with independently collected follow-up specimens. This is currently difficult as limited raw data are archived during routine assay runs. Another system, the Abbott m2000 RealTime HIV-1 assay, incorporates slope criteria (by setting criteria for the second derivative of the amplification curve, [Shain and Clemens, 2008]) in its test definition files and excludes specimens with flat slopes as being invalid. End users do not have access to assay test definition criteria and it is uncertain whether any slope criteria is applied, but the results presented here suggest that such criteria should either be included or, if already included, be more stringent in order to improve the specificity of the current assay and/or future assays. This is desirable as, apart from the cost implications of repeat testing, a delay in obtaining a definite result may delay initiation of antiretroviral therapy as soon as possible which children require to prevent HIV disease progression 
and mortality (Violari et al., 2008). It is therefore pivotal to work towards improving the current test's specificity. 


\section{Funding:}

None.

\section{Competing interests:}

The authors declare no competing interests.

\section{Ethical approval:}

The study was approved by the Research Ethics Committee of the University of Stellenbosch, N11/09/283.

\section{Acknowledgements}

We thank the diagnostic staff of the Division of Medical Virology at the National Health Laboratory Service Tygerberg for their contribution. 


\section{References}

1. Feucht UD, Forsyth B, Kruger M. 2012. False-positive HIV DNA PCR testing of infants: implications in a changing epidemic. S Afr Med J. 102(3 Pt 1):149-52.

2. Lima V, Harrigan R, Montaner JS. 2009. Increased reporting of detectable plasma HIV-1 RNA levels at the critical threshold of 50 copies per milliliter with the Taqman assay in comparison to the Amplicor assay. J Acquir Immune Defic Syndr. 1;51(1):3-6.

3. Maritz J, Preiser W, van Zyl GU. 2012. Establishing diagnostic cut-off criteria for the COBAS AmpliPrep/COBAS TaqMan HIV-1 Qualitative test through validation against the Amplicor DNA test v1.5 for infant diagnosis using dried blood spots. J Clin Virol. 53(2):106-9.

4. Moodley P, Parboosing R, Moodley D. 2013. Reduction in Perinatal HIV infections in KwaZuluNatal, South Africa in the era of more effective PMTCT Interventions (2004-2012). J Acquir Immune Defic Syndr. 63(3):410-5.

5. National Department of Health, South Africa. Guidelines on the management of HIV infection in children, $2^{\text {nd }}$ Edition 2010. Available from http://www.sahivsoc.org/practiseguidelines/national-dept-of-health-guidelines.

6. Peter Barron, Yogan Pillay, Tanya Doherty, Gayle Sherman, Debra Jackson, Sanjana Bhardwaj, Precious Robinson \& Ameena Goga. Eliminating mother-to-child HIV transmission in South Africa. Bulletin of the World Health Organization 2013;91:70-74. doi: 10.2471/BLT.12.106807.

7. Public Health Agency of Canada. HIV/AIDS Epi Updates, July 2010, Surveillance and Risk Assessment Division, Centre for Communicable Diseases and Infection Control, Public Health Agency of Canada, 2010. Obtainable from http://www.phac-aspc.gc.ca/aidssida/publication/epi/2010/index-eng.php. Accessed 17 July 2013. 
8. Public Health Ontario. CobasAmpliprep/Cobas Taqman HIV-1 Qual Test - New test for HIV1 PCR Change in Testing Methodology/Sample Submission. Labstract LAB-SD-074-000, October 2011. Obtainable from http://www.publichealthontario.ca/en/eRepository/LAB_SD_074_Cobas_amploprep_taqman_H IV1_change.pdf. Accessed 17 July 2013.

9. Roche. 2007. Roche® COBAS $\AA$ AmpliPrep/COBAS® TaqMan HIV-1 Qual Test [package insert]. Roche, Branchburg, NJ.

10. Shain EB, Clemens JM. 2008. A new method for robust quantitative and qualitative analysis of real-time PCR. Nucleic Acids Res. 36(14):e91.

11. Sherman G, Lilian R, Barron $P$, Candy S, Robinson $P$, Bhardwaj S. Laboratory information system (LIS) data is useful for monitoring the prevention of mother-to-child transmission program (PMTCT) in South Africa. In: XIX International AIDS Conference; 2012 22-27 July; Washington, United States: Abstract no. TUPE691. Available from: http://www.iasociety.org/Abstracts/A200745523.aspx. Accessed 16 July 2013.

12. Violari A, Cotton MF, Gibb DM, Babiker AG, Steyn J, Madhi SA, Jean-Philippe P, Mclntyre JA; CHER Study Team. 2008. Early antiretroviral therapy and mortality among HIV-infected infants. N Engl J Med. 359(21):2233-44.

13. Zanchetta M, Anselmi A, Vendrame D, Rampon O, Giaquinto C, Mazza A, Accapezzato D, Barnaba V, De Rossi A. 2008. Early therapy in HIV-1-infected children: effect on HIV-1 dynamics and HIV-1-specific immune response. Antivir Ther.13(1):47-55. 


\section{Tables}

Table I. Comparison between inconclusive-negative and inconclusive-positive samples.

\begin{tabular}{|c|c|c|c|}
\hline Variable & $\begin{array}{l}\text { Inconclusive- } \\
\text { negative samples }\end{array}$ & $\begin{array}{l}\text { Inconclusive- } \\
\text { positive samples }\end{array}$ & $p$ value \\
\hline Number (\%) & $193(91.5)$ & $18(8.5)$ & \\
\hline Cycle threshold $(\mathrm{Ct})$ value $^{\alpha}$ & $35.4(34.1-36.8)$ & $32.6(30.1-33.3)$ & $p<0.0001$ \\
\hline Fluorescence intensity (FI) value $^{\alpha}$ & $\begin{array}{l}0.495 \\
(0.399-0.663)\end{array}$ & $\begin{array}{l}3.326 \\
(2.064-4.370)\end{array}$ & $p<0.0001$ \\
\hline Interval between samples (days) ${ }^{\alpha}$ & $29(12-50)$ & $28(15-41)$ & NS \\
\hline Age at initial inconclusive result (days) ${ }^{\alpha}$ & $46(42-67)$ & $40(29-88)$ & NS \\
\hline $\mathrm{DBS}^{\beta}$ & $7(3.6 \%)$ & $0(0 \%)$ & - \\
\hline
\end{tabular}

${ }^{\alpha}$ Median (interquartile range), Mann-Whitney U-test

${ }^{\beta}$ number (\%)

NS = not significant 
Table II. Comparison of $\mathrm{Ct}$ and FI of initial samples as predictors of a definitive result

\begin{tabular}{|l|l|l|}
\hline Model and variable & Odds ratio $(95 \% \mathrm{Cl})$ & $\mathrm{p}$ value $(95 \% \mathrm{Cl})$ \\
\hline Univariate model - Ct & $0.655(0.542-0.791)$ & $<0.001$ \\
\hline Univariate model - FI $^{-5.348(2.939-9.732)}$ & $<0.001$ \\
\hline Adjusted model & \\
$\mathrm{Ct}$ & $1.208(0.781-1.868)$ & 0.397 \\
$\mathrm{FI}$ & $6.790(2.878-16.020)$ & $<0.001$ \\
\hline
\end{tabular}

${ }^{\alpha}$ Adjusted model included both $\mathrm{Ct}$ and FI 
Figures

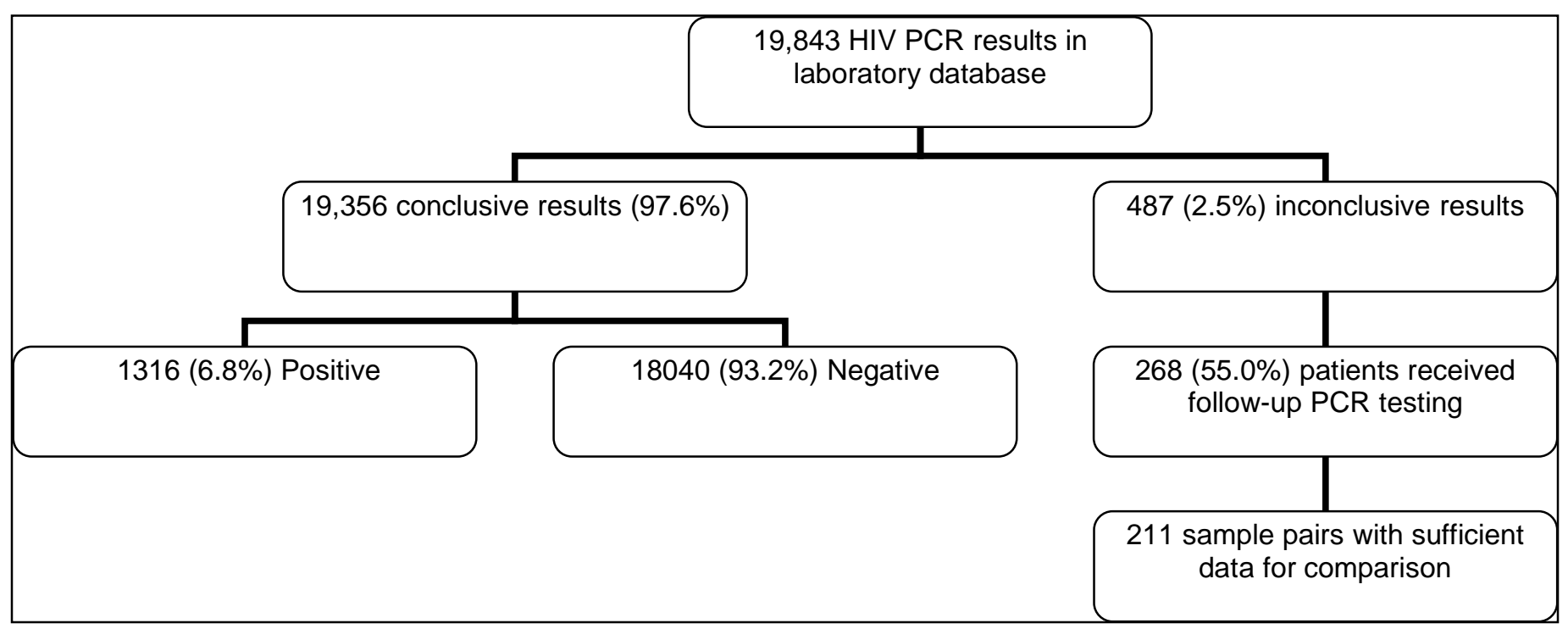

Figure I. Sample selection process. 
Measurement Details

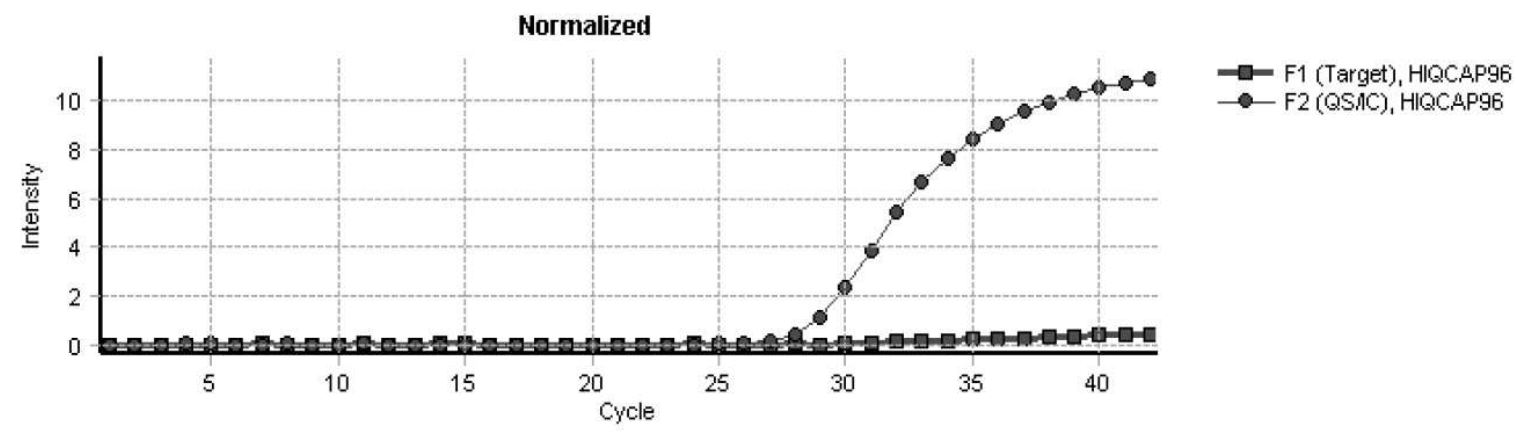

(a)

Measurement Details

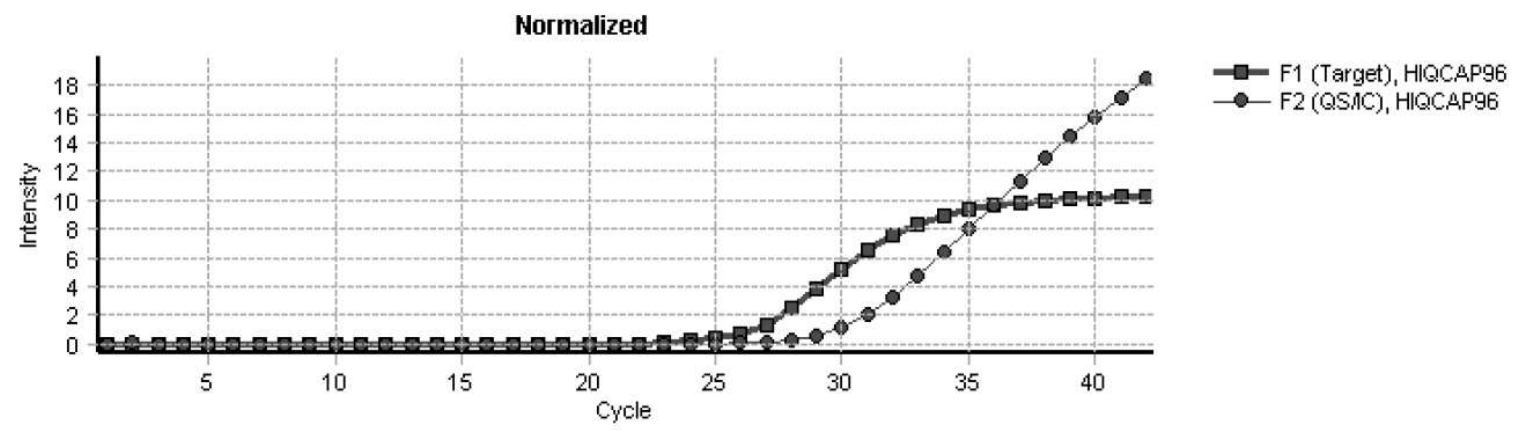

(b)

Figure II. Examples of a typical inconclusive-negative sample (a) and a positive sample (b). Amplification curves are shown as extracted from the archive of Amplilink $^{\circledR}$ software. Target amplification curves are indicated by squares and internal control amplification curves by circles. 